\title{
Quark-gluon correlations in the twist-3 TMD using light-front wave functions
}

\author{
S. Rodini* \\ Dipartimento di Fisica, Università degli Studi di Pavia, \\ INFN, Sezione di Pavia - Pavia, Italy \\ E-mail: simone.rodini01@universitadipavia.it \\ B. Pasquini \\ Dipartimento di Fisica, Università degli Studi di Pavia, \\ INFN, Sezione di Pavia - Pavia, Italy \\ E-mail: barbara.pasquini@unipv.it
}

\begin{abstract}
We discuss a T-even, twist-3 transverse momentum dependent parton distribution for unpolarized proton. We review its general, model-independent and gauge-independent decomposition, which follows from the QCD equations of motion and it is given in terms of a lower-twist distribution, a pure twist-3 distribution and singular terms. Then we present the results for the twist-3 distribution, using a Light-Front Wave Function (LFWF) overlap representation and taking into account the contribution from the three-quark and the three-quark plus a gluon proton's Fock-states. Predictions are obtained using a model for the LFWFs, based on the conformal expansion of the proton distribution amplitudes. The parameters of the LFWFs are fixed to reproduce phenomenological parametrizations of the collinear twist-2 unpolarized parton distribution.
\end{abstract}

23rd International Spin Physics Symposium - SPIN2018 -

10-14 September, 2018

Ferrara, Italy

${ }^{*}$ Speaker. 


\section{Introduction}

Twist-3 distributions are a covenient tool to study quark-gluon correlations inside the proton [1]. All the higher-twist Transverse Momentum Dependent parton distributions (TMDs) can be, in general, decomposed into different terms: lower-twist contributions, pure higher-twist contributions and singular terms. Most of the model calculations assume that the proton is a bound state of three quarks or quark-diquark bound state. One of the limitations of these types of models is that they cannot describe the "true" nature of the twist-3 distributions, related to the correlation between quarks and gluons inside the proton. Although there are no explicit gluon degrees of freedom, higher-twist effects can effectively be generated in these models from the interactions of the quarks inside the proton [2-4]. Recently, a step forward in the direction of including intrinsic gluon degrees of freedom was made in in Refs. [5-6].

In this work, after reviewing the model independent decomposition for the unpolarized twist-3 TMD, we summarize the light-front approach of Ref. [6] to model the three-quarks $(3 q)$ and three-quarks plus a gluon $(3 q+g)$ Fock-state components of the proton state and show the model results for the twist-3 distribution $e\left(x, \mathbf{k}_{\perp}\right)$.

\section{Decomposition}

Quark's TMDs are defined from the twist expansion of the following quark-quark correlator:

$$
\Phi_{i j}^{q}\left(x, \mathbf{k}_{\perp}, S\right)=\left.\int \frac{d z^{-} d \mathbf{z}_{\perp}}{(2 \pi)^{3}} e^{i z^{-} k^{+}-i \mathbf{z}_{\perp} \cdot \mathbf{k}_{\perp}}\left\langle P, S\left|\bar{\psi}_{j}(0) \mathcal{W}(0 ; z) \psi_{i}(z)\right| P, S\right\rangle\right|_{z^{+}=0} .
$$

Here and in the following, we use light-front coordinates, with $v^{ \pm}=\frac{1}{\sqrt{2}}\left(v^{0} \pm v^{3}\right)$ and $\mathbf{v}_{\perp}=\left(v^{1}, v^{2}\right)$ for a generic four-vector $v$. In Eq. $(2.1), \mathcal{W}(0 ; z)$ is a Wilson line, which ensures the gauge-invariance of the correlator. If one restrict himself to twist- 2 and twist3 TMDs for an unpolarized proton, only three distributions are present in the T-even sector: the twist-2 and chiral-even TMD $f_{1}$, the twist-3 TMDs $e$ and $f^{\perp}$, that are, respectively, chiral-odd and chiral-even. Their definition in terms of the quark-quark correlator of Eq. (2.1) is given by

$$
\begin{aligned}
& f_{1}^{q}\left(x, \mathbf{k}_{\perp}\right)=\frac{1}{2} \operatorname{Tr}\left[\Phi^{q}\left(x, \mathbf{k}_{\perp}\right) \gamma^{+}\right], \quad \frac{k_{\perp}^{j}}{P^{+}} f^{\perp, q}\left(x, \mathbf{k}_{\perp}\right)=\frac{1}{2} \operatorname{Tr}\left[\Phi^{q}\left(x, \mathbf{k}_{\perp}\right) \gamma^{j}\right] \\
& \left.\frac{M}{P^{+}} e^{q}\left(x, \mathbf{k}_{\perp}\right)=\frac{1}{2} \operatorname{Tr}\left[\Phi^{q}\left(x, \mathbf{k}_{\perp}\right) \mathbb{1}\right)\right] .
\end{aligned}
$$

If one introduces the "good" and "bad" components of the quark field as $\psi_{ \pm} \equiv \mathcal{P}^{ \pm} \psi$ where $\mathcal{P}^{ \pm}=1 / 2 \gamma^{\mp} \gamma^{ \pm}$, it turns out that the twist-2 distribution involves only good components of the quark fields, whereas the twist-3 distributions are matrix elements of the product of a good and a bad component. It can be checked from the explicit expression of the light-cone quark spinors (see, e.g., Ref.[7]), that each bad component brings a suppression factor $1 / P^{+}$with respect to a good component. In the following, we focus the attention 
only on the TMD $e^{q}$. Explicitly, the bilocal quark operator that defines the $e^{q}$ of Eq. (2.3) can be written as (here and in the following $z^{+}=0$ is understood)

$$
\mathcal{O}\left(0 ; z^{-}, \mathbf{z}_{\perp}\right) \equiv \bar{\psi}(0) \mathcal{W}(0 ; z) \psi(z)=\bar{\psi}_{+}(0) \mathcal{W}(0 ; z) \psi_{-}(z)+\bar{\psi}_{-}(0) \mathcal{W}(0 ; z) \psi_{+}(z)
$$

It is known that the bad component is not an independent dynamical field, since it is constrained by the equation of motion at fixed light-cone time:

$$
i D^{+} \psi_{-}=-\frac{\gamma^{+}}{2}\left(i \not D_{\perp}-m\right) \psi_{+},
$$

with $D^{+}$being a spatial derivative. For $k^{+}>0$, Eq. (2.5) can be easily inverted. In the case of $k^{+}=0$, we can manipulate Eq. (2.5) using a subtraction point at $0^{-}$, leading to the following operator identity:

$$
\begin{aligned}
\left.\mathcal{W}(0 ; z) \psi_{-}(z)\right|_{z^{=}=0}= & \mathcal{W}\left(0^{-}, \mathbf{0}_{\perp} ; 0^{-}, \mathbf{z}_{\perp}\right) \psi_{-}\left(0^{-}, \mathbf{z}_{\perp}\right)+ \\
& i \int_{0^{-}}^{z^{-}} d \zeta^{-} \mathcal{W}\left(0^{-}, \mathbf{0}_{\perp} ; \zeta^{-}, \mathbf{z}_{\perp}\right) \frac{\gamma^{+}}{2}\left(i \not D_{\perp}-m\right) \psi_{+}\left(\zeta^{-}, \mathbf{z}_{\perp}\right) .
\end{aligned}
$$

Using (2.6), the operator (2.4) can be decomposed as the sum of three terms:

$$
\mathcal{O}\left(0 ; z^{-}, \mathbf{z}_{\perp}\right)=\mathcal{O}_{s}+\mathcal{O}_{m}+\mathcal{O}_{\mathrm{tw} 3}
$$

with

$$
\begin{aligned}
& \mathcal{O}_{s}=\bar{\psi}(0) \mathcal{W}\left(0^{-}, \mathbf{0}_{\perp} ; 0^{-}, \mathbf{z}_{\perp}\right) \psi\left(0^{-}, \mathbf{z}_{\perp}\right), \\
& \mathcal{O}_{m}=-i m \int_{0}^{z^{-}} d \zeta^{-} \bar{\psi}_{+}(0) \mathcal{W}\left(0^{-}, \mathbf{0}_{\perp} ; \zeta^{-}, \mathbf{z}_{\perp}\right) \gamma^{+} \psi_{+}\left(\zeta^{-}, \mathbf{z}_{\perp}\right), \\
& \mathcal{O}_{\mathrm{tw} 3}=-\frac{i}{2} \int_{0}^{z^{-}} d \zeta^{-} \bar{\psi}_{+}(0) \sigma^{j+}\left[\mathcal{W}\left(0^{-}, \mathbf{0}_{\perp} ; \zeta^{-}, \mathbf{z}_{\perp}\right) \vec{D}_{\perp, j}\left(\zeta^{-}, \mathbf{z}_{\perp}\right)+\right. \\
&\left.\overleftarrow{D}_{\perp, j}^{\dagger}(0) \mathcal{W}\left(0^{-}, \mathbf{0}_{\perp} ; \zeta^{-}, \mathbf{z}_{\perp}\right)\right] \psi_{+}\left(\zeta^{-}, \mathbf{z}_{\perp}\right),
\end{aligned}
$$

where the index $j=1,2$ labels the transverse components. By inserting the operator (2.8) in the definition of $e^{q}$, we obtain the following "singular" contribution

$$
e_{s}^{q}\left(x, \mathbf{k}_{\perp}\right)=\frac{\delta(x)}{2 M} \int \frac{d \mathbf{z}_{\perp}}{(2 \pi)^{2}} e^{-i \mathbf{z}_{\perp} \cdot \mathbf{k}_{\perp}}\left\langle P\left|\bar{\psi}(0) \mathcal{W}\left(0^{-}, \mathbf{0}_{\perp} ; 0^{-}, \mathbf{z}_{\perp}\right) \psi\left(0^{-}, \mathbf{z}_{\perp}\right)\right| P\right\rangle .
$$

Following the same procedure with the operator (2.9), we obtain the "mass" contribution as a sum of a regular term and a singular term:

$$
e_{m}^{q}=\frac{m}{M x} f_{1}^{q}\left(x, \mathbf{k}_{\perp}\right)-\frac{m}{M} \delta(x) \int_{-1}^{1} d y \frac{f_{1}^{q}\left(y, \mathbf{k}_{\perp}\right)}{y} .
$$


Finally, with (2.10), we obtain the pure twist-3 contribution to $e^{q}$ :

$$
e_{\mathrm{tw} 3}^{q}\left(x, \mathbf{k}_{\perp}\right)=\tilde{e}^{q}\left(x, \mathbf{k}_{\perp}\right)-\delta(x) \int_{-1}^{1} d y \tilde{e}^{q}\left(y, \mathbf{k}_{\perp}\right)
$$

where

$$
\tilde{e}^{q}\left(x, \mathbf{k}_{\perp}\right)=-\frac{i}{M x} \Phi_{A, j}^{q,\left[\sigma^{j+}\right]}\left(x, \mathbf{k}_{\perp}\right)
$$

is defined in terms of the quark-gluon-quark correlation function [8]:

$$
\begin{aligned}
& \Phi_{A, j}^{q,\left[\sigma^{j+}\right]}\left(x, \mathbf{k}_{\perp}\right)=\frac{1}{2} \operatorname{Tr}\left[\Phi_{A, j}^{q}\left(x, \mathbf{k}_{\perp}\right) \sigma^{j+}\right]=\frac{g_{s}}{2} \int \frac{d z^{-} d \mathbf{z}_{\perp}}{2(2 \pi)^{3}} e^{i k^{+} z^{-}-\mathbf{k}_{\perp} \cdot \mathbf{z}_{\perp} \times} \\
& \left(\int_{\infty^{-}}^{\zeta^{-}} d \eta^{-}\left\langle P\left|\bar{\psi}(0) \mathcal{W}\left(0^{-}, \mathbf{0}_{\perp} ; \eta^{-}, \mathbf{z}_{\perp}\right) G_{j}^{+}\left(\eta^{-}, \mathbf{z}_{\perp}\right) \sigma^{j+} \mathcal{W}\left(\eta^{-}, \mathbf{z}_{\perp} ; \zeta^{-}, \mathbf{z}_{\perp}\right) \psi\left(\zeta^{-}, \mathbf{z}_{\perp}\right)\right| P\right\rangle+\right. \\
& \left.\int_{0^{-}}^{\infty^{-}} d \eta^{-}\left\langle P\left|\bar{\psi}(0) \mathcal{W}\left(0^{-}, \mathbf{0}_{\perp} ; \eta^{-}, \mathbf{0}_{\perp}\right) G_{j}^{+}\left(\eta^{-}, \mathbf{0}_{\perp}\right) \sigma^{j+} \mathcal{W}\left(\eta^{-}, \mathbf{0}_{\perp} ; \zeta^{-}, \mathbf{z}_{\perp}\right) \psi\left(\zeta^{-}, \mathbf{z}_{\perp}\right)\right| P\right\rangle\right) .
\end{aligned}
$$

Collecting the results of Eqs. (2.12) and (2.14) we obtain the following general decomposition for $e^{q}\left(x, \mathbf{k}_{\perp}\right)$ :

$e^{q}\left(x, \mathbf{k}_{\perp}\right)=e_{s}^{q}\left(x, \mathbf{k}_{\perp}\right)+\tilde{e}^{q}\left(x, k_{\perp}\right)+\frac{m}{x M} f_{1}^{q}\left(x, \mathbf{k}_{\perp}\right)-\delta(x) \int_{-1}^{1} d y\left(\frac{m}{M y} f_{1}^{q}\left(y, \mathbf{k}_{\perp}\right)+\tilde{e}^{q}\left(y, \mathbf{k}_{\perp}\right)\right)$.

\section{Model}

In order to calculate the pure twist-3 term directly from the expression in terms of the quark-gluon-quark correlator (see Eq. (2.14)), we need to introduce a model with intrinsic gluon contributions. We chose a framework based on the Fock-state expansion of the proton state within light-front quantization. In the light-front Fock expansion each state is weighed with a LFWF, that represents the probability amplitude of the corresponding Fock state in the proton. We truncate the expansion to the $3 q+g$ state:

$$
|P, \Lambda\rangle=|P, \Lambda\rangle_{3 q}+|P, \Lambda\rangle_{3 q+g}
$$

with

$$
\begin{aligned}
|P, \Lambda\rangle_{3 q} & =\sum_{\beta} \int[D x]_{3} \Psi_{3 q}^{\Lambda}(\beta, r) \varepsilon^{c c_{2} c_{3}} \prod_{i=1}^{3}\left|\lambda_{i}, q_{i}, c_{i}, \tilde{k}_{i}\right\rangle \\
|P, \Lambda\rangle_{3 q+g} & =\sum_{\beta} \int[D x]_{4} \Psi_{3 q+g}^{\Lambda}(\beta, r) \varepsilon^{d c_{2} c_{3}} T_{d, c}^{a}\left(\prod_{i=1}^{3}\left|\lambda_{i}, q_{i}, c_{i}, \tilde{k}_{i}\right\rangle\right)\left|\lambda_{4}, g, a, k_{i}\right\rangle .
\end{aligned}
$$

In Eqs. (3.2) and (3.3), $\Psi_{3 q}^{\Lambda}$ and $\Psi_{3 q+g}^{\Lambda}$ are, respectively, the LFWFs for the $N=3$ and $N=4$ parton Fock state $\prod_{i=1}^{N}\left|\lambda_{i}, q_{i}, c_{i}, k_{i}\right\rangle$, with $\lambda_{i}$ the parton light front helicity, $q_{i}=u, d$ and $g$ the quark and gluon flavor index, $c_{i}$ the parton color index, and $k_{i}$ the parton momentum. 

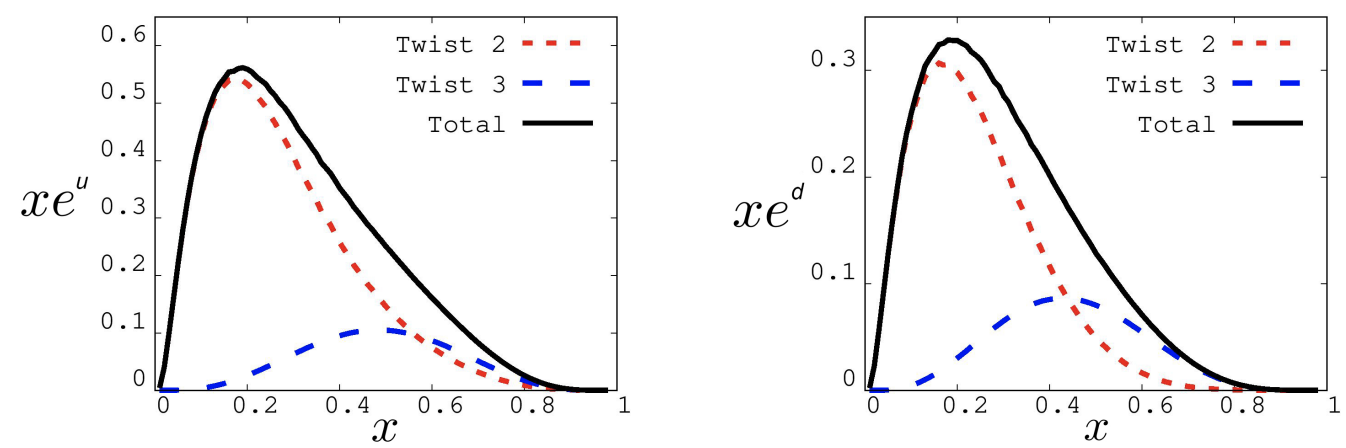

Figure 1: Results for the twist-3 distribution $x e(x)$ for the up (left panel) and down quark (right panel). The blue long-dashed curves are the pure twist-3 terms, the red short-dashed curves correspond to the mass-terms in Eq. (2.15), and the solid black curves show the total results.

For the argument of the LFWFs, we used a collective notation, with $\beta=\left(\left\{\lambda_{i}\right\} ;\left\{q_{i}\right\}\right)$ and $r=\left\{\tilde{k}_{i}\right\}$, where $\tilde{k}_{i}=\left(k_{i}^{+}=x_{i} P^{+}, \boldsymbol{k}_{\perp, i}\right)$. Furthermore, the sum over the color indexes is understood, and, then, using also the sum over the flavor indexes, the color matrix can be saturated with the color index of the first quark only.

The functional form of the LFWFs can be fixed by using the relations between the LFWFs and the Distribution Amplitudes (DAs) of the proton. The DAs can be parametrized using a polynomial expression based on the conformal expansion of the QCD, as outlined in Ref. [5]. In our work, we modified this parametrization by including non-vanishing masses for the quarks and the gluons. We fixed the parameters of the LFWFs by fitting the phenomenological parametrization of the twist-2 parton distribution $f_{1}^{q}(x)$ from Ref. [9], which provides results at the same scale of our model, i.e. $Q^{2}=1 \mathrm{GeV}^{2}$.

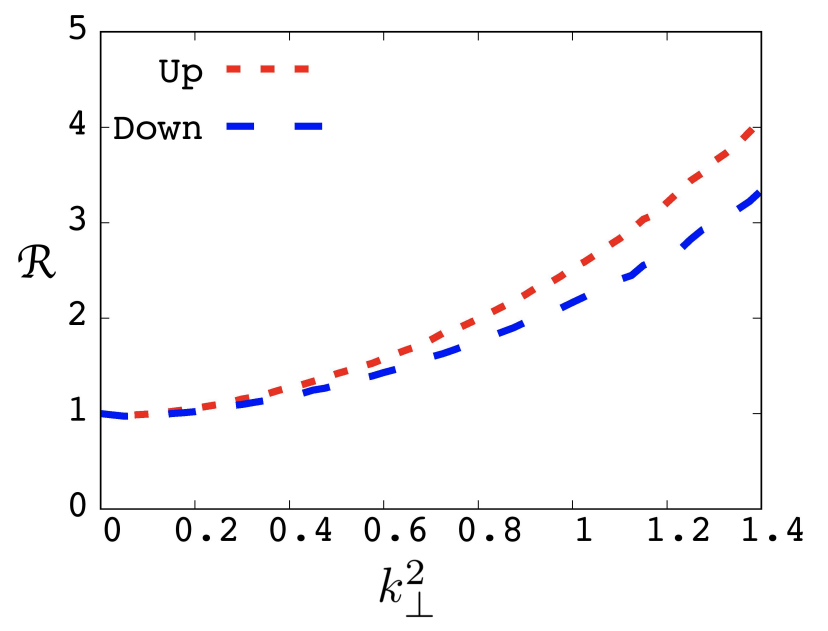

Figure 2: Ratio of the pure twist-3 term over the twist-2 distribution $f_{1}$ for the up (red line) and down quark (blue line) as function of $k_{\perp}^{2}$ and at $x=0.5$, normalized to the point $\mathbf{k}_{\perp}=\mathbf{0}_{\perp}$.

The $\tilde{e}^{q}$ distribution can be expressed as the convolution of the LFWFs for the $3 q$ state 
and the $3 q+g$ state with the same component of orbital angular momentum in the $\hat{z}$ direction. For the explicit expressions of the LFWFs overlap representation we refer to [6]. The model results for $e^{q}(x)=\int d \mathbf{k}_{\perp} e^{q}\left(x, \mathbf{k}_{\perp}\right)$ are shown in Fig. 1. One notices the nonnegligible size of the pure twist-3 contribution with respect to the mass term in Eq. (2.15), which is proportional to the twist- 2 distribution $f_{1}$. The two contributions have a quite different $x$-dependence. We also note that the pure twist- 3 contributions for the up and down quarks have very similar size, whereas the mass-term contribution for the up quark is approximately twice as large as the mass-term contribution for the down quark. The model results for the ratio

$$
\mathcal{R}=\frac{\tilde{e}^{q}\left(x=0.5, \mathbf{k}_{\perp}\right) / \tilde{e}^{q}(x=0.5,0)}{f_{1}^{q}\left(x=0.5, \mathbf{k}_{\perp}\right) / f_{1}^{q}(x=0.5,0)}
$$

are shown in Fig. 2. One can appreciate the growth in $k_{\perp}^{2}$ of the ratio, due to the different behaviour in the transverse space of the pure twist-3 distribution with respect to the twist-2 one. In particular, it is evident that the twist-3 distribution is more spread in the transverse momentum space.

\section{Acknowledgments}

This work is partially supported by the European Research Council (ERC) under the European Union's Horizon 2020 research and innovation programme (grant agreement No. 647981, 3DSPIN).

\section{References}

[1] K. Goeke, A. Metz and M. Schlegel, Parameterization of the quark-quark correlator of a spin-1/2 hadron, Phys. Lett. B618 (2005) 90 [hep-ph/0504130].

[2] H. Avakian, A. V. Efremov, P. Schweitzer and F. Yuan, The transverse momentum dependent distribution functions in the bag model, Phys. Rev. D81 (2010) 074035 [1001.5467].

[3] C. Lorcé, B. Pasquini and P. Schweitzer, Unpolarized transverse momentum dependent parton distribution functions beyond leading twist in quark models, JHEP 01 (2015) $103[1411.2550]$.

[4] C. Lorcé, B. Pasquini and P. Schweitzer, Transverse pion structure beyond leading twist in constituent models, Eur. Phys. J. C76 (2016) 415 [1605.00815].

[5] V. M. Braun, T. Lautenschlager, A. N. Manashov and B. Pirnay, Higher twist parton distributions from light-cone wave functions, Phys. Rev. D83 (2011) 094023 [1103.1269].

[6] B. Pasquini and S. Rodini, The twist-three distribution $e^{q}\left(x, k_{\perp}\right)$ in a light-front model, Phys. Lett. B788 (2019) 414 [1806.10932]. 
[7] C. Lorcé and B. Pasquini, On the Origin of Model Relations among Transverse-Momentum Dependent Parton Distributions, Phys. Rev. D84 (2011) 034039 [1104.5651].

[8] D. Boer, P. J. Mulders and F. Pijlman, Universality of T odd effects in single spin and azimuthal asymmetries, Nucl. Phys. B667 (2003) 201 [hep-ph/0303034].

[9] L. A. Harland-Lang, A. D. Martin, P. Motylinski and R. S. Thorne, Parton distributions in the LHC era: MMHT 2014 PDFs, Eur. Phys. J. C75 (2015) 204 [1412.3989]. 